\title{
Wild Photoshoot: Applying Overt and Covert Attention
}

Danny Plass-Oude Bos*, Bram van de Laar*, Matthieu Duvinage\#, Oytun Oktay@, Jaime

Delgado Saa\$, Marijn van Vliet*, Mannes Poel*, Linsey Roijendijk\%, Ali Bahramisharif\%, Boris Reuderink*

\author{
*University of Twente, The Netherlands \\ \#University of Mons, Belgium \\ @Namik Kemal University, Turkey \\ \$Sabanci University, Turkey \\ \%Radboud University Nijmegen, The Netherlands
}

We developed Wild Photoshoot, a game that uses naturally-occurring neurophysiological activity to augment the interaction in a virtual environment in an intuitive way. In this game, the user is a wildlife photographer. Besides normal movement controls (mouse and keyboard), the camera is adjusted according to where the user is looking (overt attention, $\mathrm{OA}$ ). When the animal has been found, the user will have to use covert attention (CA) (Van Gerven et al., 2009) to take the picture, because when the user looks at the animal directly, it will flee. The mental tasks for $O A$ and $C A$ come naturally given the situation.

Initial offline tests assessed the performance of EEG-based CA and EOG-based OA. For CA, the average accuracy was $67 \%$ (2 classes, 4 participants), with the pipeline: common average reference, band pass $8-14 \mathrm{~Hz}$, whitening, covariance and logistic regression. The pipeline for OA is based on Barea et al., 2003 and Itakura and Sakamoto, 2010: band pass 0.05-20 Hz, derivation, threshold, integration, and linear regression. For horizontal eye movement the average error was $2.2 \mathrm{~cm}$, and for vertical eye movement $4.8 \mathrm{~cm}$ (4 participants).

Although $\mathrm{BCls}$ are the last option for interaction for those patients who have no residual muscle control, there are also patients with limited control, who could benefit from a hybrid $\mathrm{BCl}$ setup which combines these two inputs. The naturalness of these inputs can make $\mathrm{BCls}$ easy to use; an aspect that will be appreciated by both patients and healthy users.

Van Gerven, M., Bahramisharif, A., T. Heskes, T. \& Jensen, O. (2009). Selecting features for $\mathrm{BCl}$ control based on a covert spatial attention paradigm, Neural Networks, 22:9, 12711277.

Barea. R., Boquete. L., Mazo, M., \& López, E. (2002). Wheelchair guidance strategies using EOG. JIRS. 34:3, 279-299.

Itakura, N., \& Sakamoto, K. (2010). A new method for calculating eye movement displacement from AC coupled electrooculographic signals in head mounted eye-gaze input interfaces. Biomed Signal Process Control, 5:2, 142-146. 